\title{
Use of Recharge Impulse Technology in Deep Foundations Set-up
}

\author{
Wafi Bouassida ${ }^{1, a}$ Essaieb Hamdi ${ }^{1, b}$, Mounir Bouassida ${ }^{1, c}$ \\ and Youri Kharine ${ }^{2, d}$ \\ ${ }^{1}$ Université de Tunis El Manar - Ecole Nationale d'Ingénieurs de Tunis. LR14ES03-Ingénierie \\ Géotechnique, BP 37 Le Belvédère, 1002, Tunis, Tunisia \\ ${ }^{2}$ RITA FONDATIONS, Tunisia. \\ awafy.bouassida@gmail.com, bessaieb@yahoo.fr, 'mounir.bouassida@fulbrightmail.org, \\ dkharineyouri@yandex.ru
}

Keywords: RIT piles, Electrical discharge, Frictional contact, Shape effect

\begin{abstract}
This manuscript presents the use of recharge impulse technology (RIT) in pile foundations set-up through a case study. More precisely, it discusses the performance brought by such a technique in terms of increasing the bearing capacity of deep foundation systems comparing to classical set-up processes. First, a historical overview about the technology and its contribution in referenced projects is presented. Then, a discussion about the major components behind the performance of such a technique, in pile foundation set-up, comparing to bored and driven piles is highlighted. The final section illustrates the use of RIT through a case study.
\end{abstract}

\section{Recharge Impulse Technology: A historical overview}

RIT piles (Recharge-Impulse Technology piles), are cast-in-situ piles executed by means of high electrical pulse compaction. In situ, once the drilling operation and installation of steel bars elements are carried on, an injection of a $780 \mathrm{~kg} / \mathrm{m}^{3}$ cement mortar is subjected to lateral electrical pulse compaction. Fig. 1 presents a high voltage station ensuring the transmission of electrical pulses to a conductive electrode which, in turns, transmits the shock wave to the surrounding soil. As a result of this lateral compaction, the final shape of a RIT pile is therefore similar to Frankie piles (Murthy, 2002). Hence, the diameter of such a pile is greater than the diameter of the initial drilling. In practice, the final diameter of a RIT pile could attain 3 times the diameter of the initial drilling thanks to high electrical pulse compaction. Since its appearance in 1999, RIT had participated in hundreds of projects across Russia, Germany, Singapore and Tunisia. This includes high buildings, bridges and deep excavations solutions. In terms of design, it has been proven that estimated bearing capacities according to code standards and analytical methods are much lower than real bearing capacities obtained from loading tests. Hence, it is crucial to explain the major reasons behind this gap.

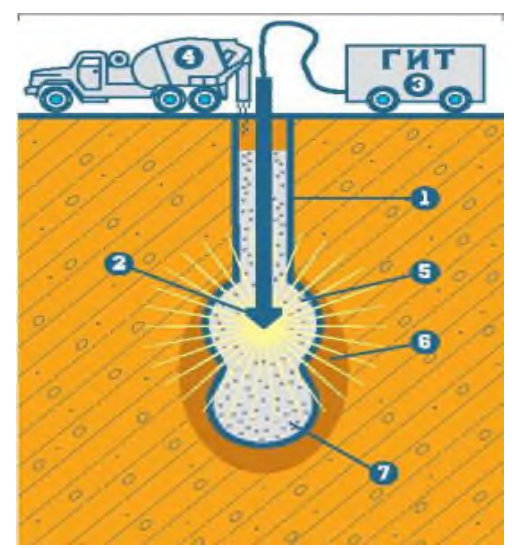

Fig. 1 Set-up process of RIT piles. 


\section{Performance of RIT Piles}

Electrical Discharge. When exerting a lateral shock wave on a soil layer, relative density increases and so on the shear strength (RITA 2008). In this context, RIT experience in saturated silty sands had shown that horizontal compaction by electrical discharge yields to an increase of both friction angle and cohesion (RITA 2008). Similar applications confirmed such a statement, peculiarly in dynamic compaction of silty soils to prevent liquefaction risks (Holtz 2000). Thus, frictional contact between a RIT pile and a set of surrounding soil layers is clearly more important than that of a bored circular pile and the same horizons due to the electrical discharge effect. This naturally yields to an increase in shaft bearing capacity of a RIT pile regarding bored piles. Compared to driven piles which are also characterized by a vibration task during the set-up process, recharge impulse technology differs in two main points. The first one is the compaction task which is done at constant volume during RIT piles installation contrary to pile driving which allows heave during the set-up process (Poulos and Davis 1980) and therefore volume change. The second point is related to the type of compaction itself. Indeed, the dynamic loading force transmitted to surrounding soil layers is not the same. For driven piles, the vibration transmitted to surrounding layers could be modeled as a classical wave producing pore pressure dissipation. Conversely, the electrical discharge, which can be modeled as an impulse, does not produce pore pressure dissipation as the duration of such a blasting load is very short (An et al 2010). Therefore, it should be noted that, although driving piles and executing RIT foundations yield to a densification of surrounding soil layers and therefore an improvement of shaft bearing capacity, the two set-up processes differ and do not reproduce the same effect in terms of surrounding layers improvement (increase in relative density ) and length of influence zones. Nowadays, as pile design codes do not propose recommendations for modeling the effect of blasting loads on surrounding soil layers and therefore on pile bearing capacities, it is natural to find a gap between in situ load capacities of RIT piles and computed ones according to such standards. Hence, it should be stated that an accurate modeling of the electrical discharge task brings us closer to RIT piles behavior and, consequently, allows a more realistic estimation of RIT load capacities.

Shape Effect. As the final shape of an installed RIT pile is not cylindrical but constituted by superposed bulbs, it's clear that the frictional mechanism developed when loading it differs from which developed when loading a cylindrical pile. Indeed, when applying a vertical force on a cylindrical pile, the frictional contact along its interface is governed at yield, by:

$$
\tau=c a+\sigma^{\prime} \tan (\delta)
$$

$\tau$ is the shear stress at yield,

$\sigma$ ': the effective vertical stress

ca: pile soil adhesion (lower than soil cohesion, (Poulos and Davis 1980))

$\delta=\frac{1}{3} \varphi$ Where $\varphi$ is the soil friction angle (Pucker et al. 2012)

Hence the shear stress along the interface is characterized by a friction angle $\delta$ lower than $\varphi$ and a pile soil adhesion lower than the surrounding soil cohesion. For the case of RIT piles, and without considering the installation effect (dynamic lateral compaction) improving both the friction and cohesion of the surrounding medium, the confined zones between the bulbs of a RIT pile, do not develop a shear stress along the pile interface during the loading. Indeed, the frictional mechanism generated during the loading task will principally be developed at the contact boundaries (CB) of these zones, with the surrounding soil and not with the pile interface (Fig. 2). Such a mechanism, produces therefore a vertical shear plan governed at yield by the friction angle and the cohesion of the surrounding soil. Hence the shear stress mobilized at yield during the vertical pile loading can be written as:

$$
\tau=c+\sigma \tan (\varphi)
$$

c: Soil cohesion 


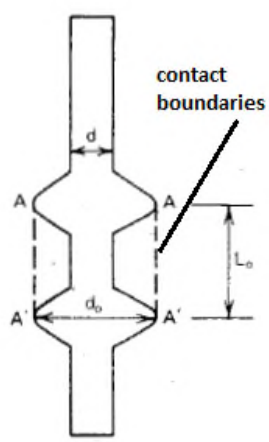

Fig. 2 Frictional mechanism developed when loading a RIT pile.

Consequently, and according to expressions (a) and (b) the frictional contact between a RIT pile and a set of surrounding soils is more important than which of a cylindrical pile and the same soil layers due to the curvy shape of the RIT pile. Such a frictional mechanism, developed when loading a RIT pile, is similar to the frictional contact of under reamed piles, composed by bulbs, and widely used in India (Mohan 1977, Poulos and Davis 1980). Indeed, pull-out in situ tests carried by Mohan (1977) on such a pile class had confirmed that the frictional mechanism related to a given pile composed by bulbs, is developed during the loading task, along the cylinder encountering the bulbs. Consequently, the shear stress distribution will be governed by friction angles and cohesions of surrounding soil layers $(\mathrm{C}, \varphi)$ and not the shear parameters characterizing the frictional contact between cylindrical piles and the surrounding soil layers (ca, $\delta$ ). Hence, and similarly to the electrical discharge, it should be stated that the curvy shape of a RIT pile is a major parameter increasing its shaft bearing capacity comparing to cylindrical piles. In the following, we will investigate the contribution of these two parameters in the performance of RIT piles through a case study.

\section{Case Study}

For the construction of a moderate high building ( 7 floors), the foundation solution was decided to be a raft resting on 22.5 meters length RIT piles having a mean diameter of 0.4 meter. Table 1 and Fig. 3 present the characteristics of the soil profile in which the predicted RIT piles will be installed.

Table 1. Cohesion and friction angle of crossed layers.

\begin{tabular}{|c|c|c|}
\hline Layer & Thickness $(\mathrm{m})$ & $\left(\right.$ Cohesion, friction) $\left(\mathrm{kPa},{ }^{\circ}\right)$ \\
\hline Clayey sand & 3 & 25,20 \\
\hline Grey carbonate clay & 10 & 35,4 \\
\hline Silty brown clay & 2.5 & 35,4 \\
\hline Grey compressible clay & 27 & 30,4 \\
\hline
\end{tabular}




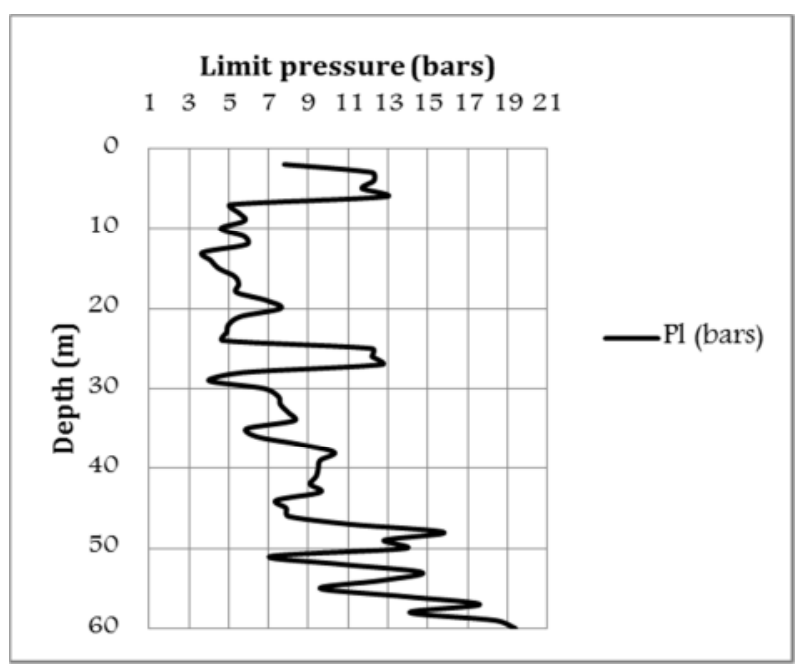

Fig. 3 Limit Pressure variation in depth.

Based on Fig. 3 data, and according to pressuremeter standards the service load capacity equals 890 KN. Similarly, based on Table 1 data and following NAVFAC DM 7.2 (1984), load capacity equals $910 \mathrm{KN}$. Fig. 4 presents two plots: The first corresponds to the results of a vertical loading test carried on a RIT pile $(\mathrm{L}=22.5 \mathrm{~m}, \mathrm{D}=0.4 \mathrm{~m})$ installed in the investigated site. The second one shows the results of a numerical model simulating a loading test (Plaxis 2D) in the same soil horizons but on bored cylindrical pile having the same dimensions of the tested RIT pile.

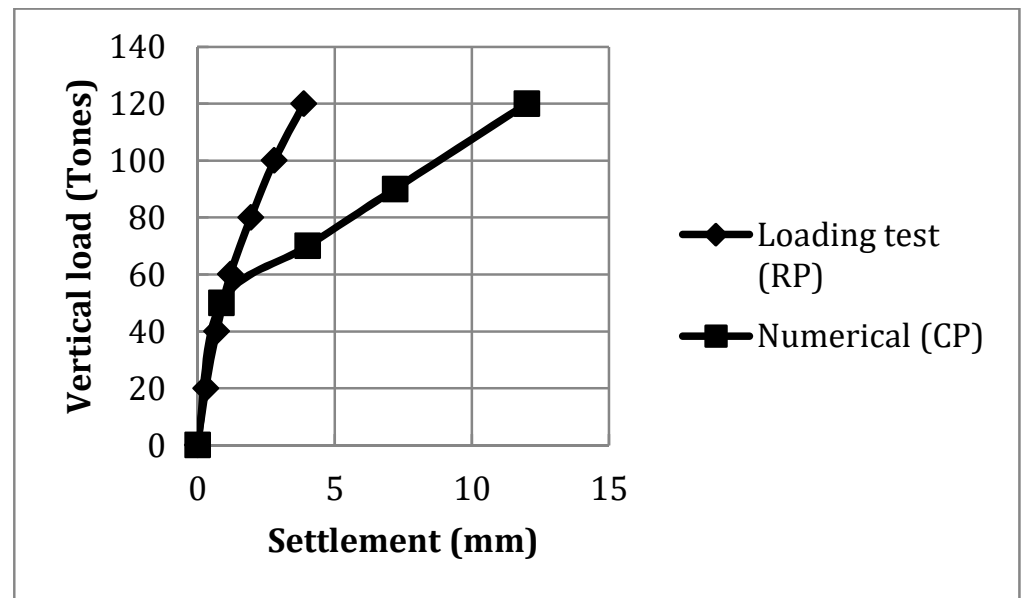

Fig. 4 Comparison between the behavior of a single RIT pile and a bored cylindrical pile installed in the same soil horizons.

According to the results plotted in Fig. 4, it is clear that the load capacity of the installed RIT pile should equal at minimum $1200 \mathrm{KN}$ as this force corresponds to 3.8 millimeters of settlement that is lower than $1 \%$ of the diameter length (Murthy, 2002). Hence, standardized codes, particularly the pressuremeter method and NAVFAC (1984) clearly underestimate the load capacity. The gap between estimated load capacities and the experimental one is primary due to the omission of the electrical discharge task and the curvy shape of RIT piles as it has been discussed in the previous section. Indeed, the frictional performance of RIT piles is clearly exposed from the difference between the two load settlement curves shown in Fig. 4. More precisely, it's clear that the shear strength has been fully mobilized along the pile-soil interface for the case of the cylindrical pile above $600 \mathrm{KN}$ due to the drastic slope change of the load-settlement curve. However, the shear strength along the RIT pile interface appears to be partially mobilized even at $1200 \mathrm{KN}$ without a significant change in the slope of its load settlement curve. Such an interpretation confirms the frictional performance of RIT piles comparing to cylindrical piles. In order to confirm the electrical discharge contribution to such a frictional performance, a pressuremeter test had been conducted in 
the investigated site, 7 months after the setup of the building foundation system, near an installed RIT pile to investigate the influence of installation on surrounding soil parameters. Fig. 5, presents the related results.

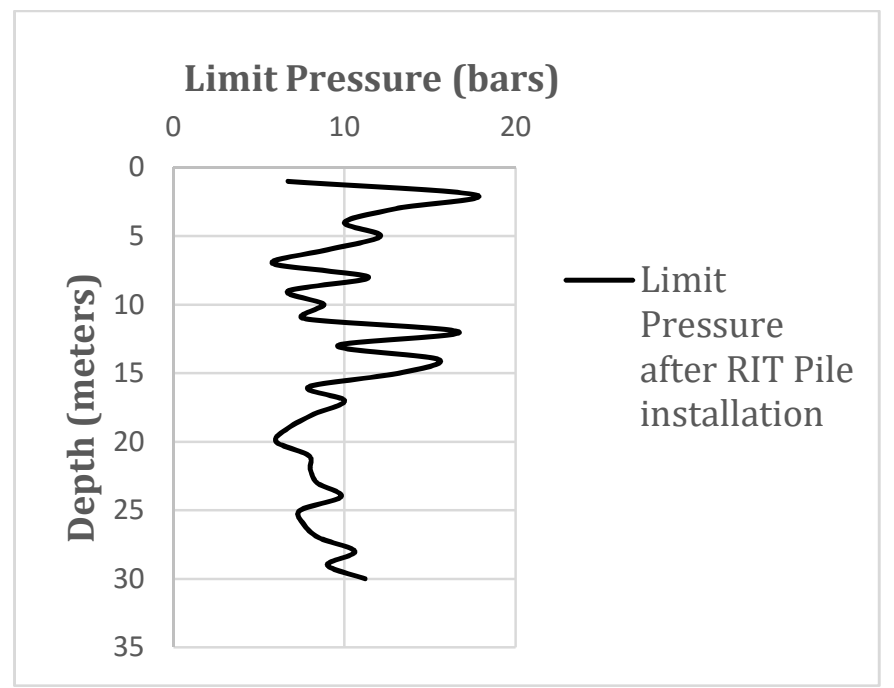

Fig. 5 Limit pressure profile after the installation of RIT piles.

Based on the obtained profile, the influence of the electrical discharge task is clearly shown. Indeed, the limit pressure profile had been improved by more than $30 \%$ comparing to the initial profile presented in Fig. 2. More precisely, estimating the load capacity according to this profile yields to a service force of $1220 \mathrm{KN}$. This therefore, validates the important contribution of the electrical discharge task to enhance the frictional bearing capacity of RIT piles comparing to cylindrical piles.

\section{Conclusion}

Classical standardized codes underestimate the bearing capacities of RIT piles as there is no account for the electrical discharge task and the curvy shape effects. Further works should axially turn around these two components for a more realistic modeling of RIT piles behavior. Indeed, from the case study presented herein, the omission of these two parameters produced an underestimation of more than $30 \%$ of the real load capacity.

\section{References}

[1] V. N. S. Murthy, Geotechnical Engineering. Taylor \& Francis Inc. Volume I: 1056 pages. NY, USA, (2002).

[2] Recharge Impulse Technologies and Apparatus, Building on the basement of knowledge. RITA Press. Volume I: 58 pages. Moscow, (2005).

[3] H. G. Poulos, E. H. Davis, Pile Foundations Analysis and Design. Jhon Wiley and Sons. 1 (1980) 397.

[4] J. An, C. Y. Tuan, F. ASCE, B. A. Cheeseman and G. A Gazonas, Simulation of Soil Behavior under Blast Loading. Int. J. Geomech. 11(4) (2011) 323-334.

[5] T. Pucker and J. Grabe, Numerical Simulation of the Installation Process of Full Displacement Piles. Comput. Geotech. 45 (2012) 93-106. 